\title{
Correlations between walking speed and Timed Up and Go (TUG) in patients with heart failure class II-III NYHA
}

\author{
Diana TURCU ${ }^{1,2}$, Anca-loana ROMAN ${ }^{3}$, Matei TEODORESCU4 ${ }^{4}$, Marius POPESCU 4 , \\ Rodica PLOESTEANU ${ }^{5}$, Liviu GHILENCEA ${ }^{4,6}$, Nicolae GICA ${ }^{6,7}$, Vlad BATAILA ${ }^{8}$, Mihai BERTEANU ${ }^{4,6}$ \\ ${ }^{1}$ Department of Cardiology, Railway General Hospital, Ploiesti, Romania \\ 2Doctoral School, "Carol Davila" University of Medicine and Pharmacy, Bucharest, Romania \\ 3"Dr. Gavril Curteanu" Clinical Hospital, Oradea, Romania \\ ${ }^{4}$ Elias Clinical Hospital, Bucharest, Romania \\ 5"Sf. Pantelimon" Clinical Hospital, Bucharest, Romania \\ 6"Carol Davila" University of Medicine and Pharmacy, Bucharest, Romania \\ ${ }^{7}$ Filantropia Clinical Hospital, Bucharest, Romania \\ ${ }^{8}$ Floreasca Clinical Hospital, Bucharest, Romania
}

\begin{abstract}
Physical reabilitation programs for patients with heart failure (HF) are underdeveloped in our contry. In order to establish the correct reahabilitation plan, the first step is to evaluate the patient's functional capacity. G-Walk is a simple and effective test in assesing functional capacity. The aim of the study is to analyze the correlation between the results obtained in Timed Up and Go (TUG) and walking speed test and the prognosis of patients with heart failure.

Material and methods. We prospectively analyzed a group of 44 patients with heart failure class II-III NYHA hospitalized in the Cardiology Department of Elias Hospital between June 17 and July 15, 2019.We evaluated the functional mobility of the patients with heart failure with the G-Walk device that contains a wireless sensor. The G-Walk device represents a gait analysis system, performing - according to a protocol -2 tests: Walk (patient's walking speed) and TUG (measures balance and mobility).

Results. After analyzing the walking speed, the patients were divided into 2 groups: those with a speed $<0.8 \mathrm{~m} / \mathrm{s}$ and those with a speed $>0.8 \mathrm{~m} / \mathrm{s}$.

Out of 44 patients, 21 patients had a walking speed $<0.8 \mathrm{~m} / \mathrm{s}$ and 23 patients had a walking speed $>0.8$ $\mathrm{m} / \mathrm{s}$. It was seen that patients with lower walking speed $(V<0.8 \mathrm{~m} / \mathrm{s})$ versus patients with better walking speed $(V>0.8 \mathrm{~m} / \mathrm{s})$ were older, had a lower body weight, a lower blood pressure value, a lower value of the oxygen saturation, a lower value of sodium and a higher blood glucose value.

After analyzing the value of the TUG test, patients were divided into 2 groups: patients with a TUG test duration $<15$ seconds and patients with a TUG test duration $>15$ seconds. Thus, out of 44 patients studied, 19 patients had a TUG value $<15$ seconds and 25 patients had a TUG value over 15 seconds.

It was observed that patients with a higher TUG value (> 15 seconds) versus patients with normal TUG value ( $<15$ seconds) were older, had a higher creatinine value and a higher blood glucose value.

Conclusions. It has been shown that a low value of walking speed and mobility is associated with increased morbity and mortality of all causes in elderly patients with cardiovascular disease. The physical rehabilitation of patients with heart failure is very important for improving the quality of life.
\end{abstract}

Keywords: functional capacity, heart failure, gait, TUG 


\section{INTRODUCTION}

The prevalence of the HF is approximately $2 \%$ of the adult population worldwide, rising to approximately $10 \%$ among people above 70 years of age.

Heart failure is associated with great economic burden on the health systems and is dependent on the frequency of hospital admissions and the duration of the admissions (1).

Heart failure is a clinical syndrome characterized by decreased ability of the heart to provide the proper amount of blood, containing nutrients and oxygen, needed by the body (2).

It is a condition that decreases the quality of life mainly by decreasing the functional capacity, which requires a correct management of the disease in order to establish a physical rehabilitation program.

Reduced functional capacity in patients with $\mathrm{HF}$ is associated with a worse prognosis and has been the main focus of many interventional treatment modalities (3).

A very important objective of rehabilitation is to improve the functional capacity of the patient in order to perform daily activities. Functional capacity measurement can be performed by several methods, including field tests, monitoring of regular activities and cardio-respiratory exercise test.

Heart failure was defined by the current symptoms of heart failure in the presence of reduced left ventricular (LV) systolic function on echocardiography and in the absence of any other cause that may reduce the LVS function $(3,4)$.

The cardio-respiratory effort test is the gold standard in assessing the functional capacity of patients but is an expensive method that requires specialized equipment and personnel.

The 6-minute walk test is an additional method in assessing the functional exercise capacity of patients with heart failure, having an important role as a predictor of mortality. It has been shown that a low value of the distance traveled in the 6-minute walk test in patients with heart failure is associated with an increase in total mortality and an increase in the rate of respitalization (5).

The test requires a plot of land of at least $25 \mathrm{~m}$, which is not always possible in a hospital.

Given the need for a simple and efficient evaluation, in recent years there has been an attempt to develop different methods for assessing the functional capacity of patients.

G-Walk is a system for the functional analysis of movement and introduces a new approach to motion analysis: a specialized wireless sensor is applied to the patient and enables him to perform clinical tests such as the TUG and the 6 minutes walking test very quickly.
This evaluation is a tool for helping physicians and specialists in the field of rehabilitation to assess the patient special conditions and evaluate the efficacy of treatments and rehabilitation therapies.

The protocols allow us to carry out complete analyses of the main clinical tests: Timed Up and Go - assessment of functional mobility and fall risk, six minutes walking test - evaluation of the functional capacity of patients of any age, walk, turn test, run and jumps.

\section{METHODS}

We conducted a prospective study between June 15 and July 17, 2019, where we analyzed 44 patients with NYHA class II-III heart failure, being evaluated using the G-Walk device to determine their mobility. Patients were hospitalized in the Cardiology Clinic for the treatment of heart failure.

Each patient was explained the methodology of the study and a handwritten signed consent was obtained from each patient who wished to enroll in the study.

We set out to conduct this study given the fact that heart failure is a disease with a high prevalence in the elderly, which develops decreased mobility, ultimately leading to decreased quality of life and increased morbidity and mortality.

Patients were divided into two groups, depending on the results obtained from the evaluation by G-Walk: patients with low walking speed $<0.8 \mathrm{~m} / \mathrm{s}$ versus patients with normal walking speed $>0.8 \mathrm{~m} / \mathrm{s}$ and patients with TUG $>15 \mathrm{~s}$ versus patients with normal TUG values $<15 \mathrm{~s}$

Inclusion criteria: ICC class II-III NYHA, retained mobility, minimum age over 18 years and informed consent signed.

Exclusion criteria: acute myocardial infarction, pulmonary thromboembolism, neoplasms, history of stroke, immobilized patients.

The initial assessment included anamnestic data on medical history, risk factors, clinical examination, drug treatment at home. All patients underwent echocardiography, EKG and had a complete set of blood tests.

Monitored parameters: walking speed, Timed UP and Go, sex, weight, age, presence/absence of smoking, oxygen saturation, echocardiographic data, biological parameters (hemoglobin, creatinine, $\mathrm{Na}, \mathrm{K}$, blood sugar), personal pathological history (hypertension, diabetes, ischemic heart disease, coronary revascularization, atrial fibrillation).

The parameters were determined using the G-Walk device, an echocardiograph from the cardiology department and the medical analysis laboratory at Elias University Emergency Hospital.

The cardiac ultrasound parameters evaluated were: systolic function of the left and the right ventricle, the 
dimensions of the cardiac cavities, presence/absence of valvopathies and pulmonary hypertension.

We evaluated the functional mobility with the G-Walk device that contains a wireless sensor. The G-Walk device represents a gait analysis system, performing- according to a protocol- 2 tests: Walk and TUG.

The Walk test recorded the patient's walking speed over a distance of $2 \times 6$ meters, in an area arranged on the hospital corridor. The patients were divided into two groups: those with a walking speed $<0.8 \mathrm{~m} / \mathrm{s}$ (group A), and those with a walinkg speed above 0.8 $\mathrm{m} / \mathrm{s}$ (group $B$ ).

Patients were instructed to walk as far as possible, turning $180^{\circ}$ every $12 \mathrm{~m}$ in the allotted time of $6 \mathrm{~min}$. A low walking speed was considered to be a value $<0.8$ $\mathrm{m} / \mathrm{s}$.

The Timed Up and Go (TUG) test is a functional test used to measure the balance and mobility in reabilita- tion centers (6). The patients were divided into two groups: those with TUG < 15 s (group A), and those with TUG above $15 \mathrm{~s}$ (group B).

The patients were placed on a chair, asked to stand up, walk 3 meters and return to the chair at his normal pace.

The results of the TUG test were evaluated as follows: normal mobility - TUG < 15 s, slightly low mobility - TUG 15-25 s, moderately low mobility - TUG > 25 s, severely impaired mobility - patients who failed to complete the test.

\section{Statistical analysis}

The database was compiled in the MS Access 2007 program and then exported and processed initially in Excel. Subsequently, after adjusting and preparing all variables, they were introduced in SPSS. The SPSS variant used was 22.0.

TABLE 1. The analysis of baseline descriptive statistical differences for the discrete variables of the two groups

\begin{tabular}{|c|c|c|}
\hline & $\begin{array}{l}\text { Lot A speed }<0.8 \mathrm{~m} / \mathrm{s} \\
(\mathrm{n}=21)\end{array}$ & $\begin{array}{l}\text { Lot B speed }>0.8 \mathrm{~m} / \mathrm{s} \\
(\mathrm{n}=23)\end{array}$ \\
\hline Female gender $\mathrm{n} \%$ & $12(57.1 \%)$ & $7(30.4 \%)$ \\
\hline Smokers n\% & $2(9.5 \%)$ & $11(47.8 \%)$ \\
\hline Arterial hypertension\% & $19(90.5 \%)$ & $16(69.6 \%)$ \\
\hline Dyslipidemia, n\% & $18(85.7 \%)$ & $20(87 \%)$ \\
\hline Diabetes mellitus, $\mathrm{n} \%$ & $6(28.6 \%)$ & $4(17.4 \%)$ \\
\hline History of heart failure, $\mathrm{n} \%$ & $21(100 \%)$ & $15(65.2 \%)$ \\
\hline History of atrial fibrillation $\mathrm{n} \%$ & $13(61.9 \%)$ & $12(52.2 \%)$ \\
\hline History of ischemic coronary artery disease, $n \%$ & $14(66.7 \%)$ & $12(52.2 \%)$ \\
\hline History of revascularization $\mathrm{n} \%$ & $7(33.3 \%)$ & $5(21.7 \%)$ \\
\hline Furosemide, $\mathrm{n} \%$ & $16(76.2 \%)$ & $14(60.9 \%)$ \\
\hline Spironolactone, n\% & $10(47.6 \%)$ & $6(26.1 \%)$ \\
\hline Digoxin, $\mathrm{n} \%$ & $6(28.6 \%)$ & $3(13 \%)$ \\
\hline Angiotensin-converting enzyme inhibitor, $\mathrm{n} \%$ & $14(66.7 \%)$ & $12(52.2 \%)$ \\
\hline Double antiplatelet therapy, $\mathrm{n} \%$ & $6(28.6 \%)$ & $1(4.3 \%)$ \\
\hline Betablocker, $\mathrm{n} \%$ & $17(81.0 \%)$ & $15(65.2 \%)$ \\
\hline Oral anticoagulation, $\mathrm{n} \%$ & $12(57.1 \%)$ & $9(39.1 \%)$ \\
\hline Age, standard deviation (SD) & $71.81(6.266)$ & $66.78(12.982)$ \\
\hline NTproBNP, SD & $4138.50(4186.760)$ & 4739.78(5118.982) \\
\hline Weight, SD & $72.57(13.457)$ & $87.39(25.464)$ \\
\hline Heart rate, SD & $88.43(30.498)$ & $88.04(28.049)$ \\
\hline Arterial tension, SD & $116.19(21.266)$ & $134.87(25.742)$ \\
\hline Sat $\mathrm{O}_{2}, \mathrm{SD}$ & $94.62(2.224)$ & $95.00(3.205)$ \\
\hline Right ventricle, SD & $33.65(9.074)$ & $36.17(8.283)$ \\
\hline Left atrium, SD & $43.37(7.388)$ & $46.04(8.127)$ \\
\hline Diastolic transverse diameter of the left ventricle, SD & $50.70(9.325)$ & $53.87(8.120)$ \\
\hline Tricuspid annular presystolic excursion, SD & $18.47(3.717)$ & $18.52(4.986)$ \\
\hline Hemoglobin, SD & $12.106(1.5865)$ & $12.952(1.7699)$ \\
\hline Creatinine, SD & $1.2382(0.70965)$ & $1.2130(0.50727)$ \\
\hline $\mathrm{Na}, \mathrm{SD}$ & 136.22(4.989) & 138.35(3.113) \\
\hline K, SD & $4.5(0.6869)$ & $4.509(0.6653)$ \\
\hline Glycemia, SD & $115.50(54.735)$ & $107.82(27.114)$ \\
\hline
\end{tabular}


Given the relatively small batch, but with completely independent records, we considered that only the usual methods of verifying the normal distribution of the studied parameters are needed. The methods of testing the normality of the data are, as the case may be, the evaluation of the histogram of the data, determining the mean, median, standard deviation, standard error and implicitly rule 68-95-99.7 as well as Kolmogorov Smirnov testing.

The studied parameters are represented by continuous variables and categorical variables, mostly binary. Continuous variables were compared using the twotailed student T-test, a test that uses the comparison of the means of the two variables and the standard deviation with the $\mathrm{F}$ or Levene test.

For the evaluation of the correlated results at hospitalization and discharge for the paired continuous variables, the paired T-test was used. The binary variables were compared using the chi-square test, either automatically or initially forming contingency tables, the significance of the variations being obtained from the ratio of observed and expected values. For variables that do not follow the normal or $Z$ distribution or for those with low values, nonparametric tests, the Wilkoxon rank-sum test for independent continuous variables or the Fischer test exactly for independent categorical variables will be used. Given values frequently below 10, the exact Fischer test was predominantly used.

\section{RESULTS}

Our study group consisted of 44 patients with a mean age of $62.7+/-12.1$. The average length of hospitalization was 9.3 days. The average walking speed of the whole group was $0.8416 \mathrm{~m} / \mathrm{s}$ (SD 0.18890). The average discharge speed of the whole group was 0.8820 $\mathrm{m} / \mathrm{s}$ (SD 0.23887).

After analyzing the walking speed, the patients were divided into 2 groups: those with a speed $<0.8$ $\mathrm{m} / \mathrm{s}$ and those with a speed $>0.8 \mathrm{~m} / \mathrm{s}$. Out of $44 \mathrm{pa}-$ tients, 21 patients had a walking speed $<0.8 \mathrm{~m} / \mathrm{s}$ and 23 patients had a walking speed $>0.8 \mathrm{~m} / \mathrm{s}$.

TABLE 2. Analysis of baseline descriptive statistical differences for the discrete variables of the two groups

\begin{tabular}{|c|c|c|}
\hline & $\begin{array}{c}\text { Lot } A, \text { TUG < 15s } \\
(n=19)\end{array}$ & $\begin{array}{c}\text { Lot } B, \text { TUG }>15 s \\
(n=25)\end{array}$ \\
\hline Female gender $n \%$ & $7(36.8 \%)$ & $12(48 \%)$ \\
\hline Smokers, n\% & $6(31.6 \%)$ & $7(28 \%)$ \\
\hline Arterial hypertension, $\mathrm{n} \%$ & $15(78.9 \%)$ & $20(80.0 \%)$ \\
\hline Dyslipidemia, n\% & $18(85.7 \%)$ & $20(87 \%)$ \\
\hline Diabetes mellitus, $\mathrm{n} \%$ & $4(21.1 \%)$ & $6(24 \%)$ \\
\hline History of atrial fibrillation, $\mathrm{n} \%$ & $9(47.4 \%)$ & $16(64.0 \%)$ \\
\hline History of ischemic coronary disease, $n \%$ & $10(52.6 \%)$ & $16(64 \%)$ \\
\hline History of revascularization, $n \%$ & $4(21.1 \%)$ & $8(32 \%)$ \\
\hline Furosemide - pre, $\mathrm{n} \%$ & $12(63.2 \%)$ & $18(72 \%)$ \\
\hline Spironolactone, $\mathrm{n} \%$ & $5(26.3 \%)$ & $11(44 \%)$ \\
\hline Digoxin, $n \%$ & $2(10.5 \%)$ & $7(28 \%)$ \\
\hline Angiotensin-converting enzyme inhibitor, n\% & $9(47.4 \%)$ & $17(68 \%)$ \\
\hline Double antiplatelet therapy, $\mathrm{n} \%$ & $0(0 \%)$ & $7(28 \%)$ \\
\hline Betablocker, n\% & $12(63.2 \%)$ & $20(80 \%)$ \\
\hline Oral anticoagulation, $\mathrm{n} \%$ & $6(31.6 \%)$ & $15(60 \%)$ \\
\hline Age, SD & $66.89(11.362)$ & $70.92(9.729)$ \\
\hline NTproBNP, at admission, SD & 4563.75 (4032.289) & $4398.50(5035.113)$ \\
\hline Weight at admission, SD & $81.32(23.048)$ & $79.56(21.115)$ \\
\hline Heart rate, at admission, SD & $92.11(30.127)$ & $85.28(28.191)$ \\
\hline Arterial tension, at admission, SD & $134.84(27.817)$ & $119.20(21.296)$ \\
\hline Sat $\mathrm{O}_{2}$ at admission, $\mathrm{SD}$ & $94.63(3.320)$ & $94.96(2.300)$ \\
\hline Right ventricle diameter, SD & 34.89 (10.049) & $35.08(7.587)$ \\
\hline Left atrium, SD & $46.79(8.290)$ & $43.22(7.198)$ \\
\hline Diastolic transverse diameter of the left ventricle, SD & $52.21(8.702)$ & $52.54(8.959)$ \\
\hline Tricuspid annular presystolic excursion, SD & $18.74(4.280)$ & $18.29(4.551)$ \\
\hline Hemoglobin, SD & $12.518(1.8331)$ & $12.625(1.6819)$ \\
\hline Creatinine, SD & $1.0618(.33332)$ & $1.3435(.71260)$ \\
\hline $\mathrm{Na}, \mathrm{SD}$ & $137.28(3.159)$ & $137.52(4.823)$ \\
\hline $\mathrm{K}, \mathrm{SD}$ & $4.539(.6260)$ & $4.478(.7090)$ \\
\hline Glycemia, SD & $106.87(26.686)$ & $115.44(52.041)$ \\
\hline
\end{tabular}


It was seen that patients with lower walking speed $(\mathrm{V}<0.8 \mathrm{~m} / \mathrm{s})$ versus patients with better walking speed ( $\mathrm{V}>0.8 \mathrm{~m} / \mathrm{s})$ were older $(71.81+/-6.26$ years vs. 66.78 $+/-12.98 \mathrm{p}=0.10)$, had a lower body weight (72.57 +/- 13.457 vs. $87.39+/-25.464 p=0.02)$, a lower blood pressure value (116.19+/- 21.266 vs. $134.87+/-25.742$ $p=0.12)$, a lower value of the oxygen saturation (94.62 $+/-2.224$ vs. $95.00+/-3.205 p=0.647)$, a lower value of $\mathrm{Na}(136.22+/-4.989$ vs. $138.35+/-3.113 p=0.102))$ and a higher blood glucose value (115.50 +/- 54.735 vs. $107.82+/-27.114 p=0.615)$. Also in the group of patients with walking speed $>0.8 \mathrm{~m} / \mathrm{s}$ the history of chronic HF was $65.2 \%$ compared to the group of patients with low walking speed where the history of chronic HF was $100 \%$.

Regarding the association between walking speed and the risk factors of the patients, it was observed that in the group of patients with $\mathrm{V}<0.8 \mathrm{~m} / \mathrm{s}: 57.1 \%$ were women, $90.5 \%$ were hypertensive, $85.7 \%$ dyslipidemic, $28.6 \%$ had type II diabetes, $100 \%$ had a history of chronic HF, $61.9 \%$ had a history of atrial fibrilation and $66.7 \%$ had a history of ischemic coronary disease. Walking speed was significantly improved after treatment for heart failure.

The average value of the TUG test at the hospitalization of the whole group was 16.7193 seconds (SD 5.56735). The average value of the TUG test at discharge of the whole group was 16.7891 seconds (SD 4.87219).

After analyzing the value of the TUG test, patients were divided into 2 groups: patients with a TUG test duration $<15$ seconds and patients with a TUG test duration $>15$ seconds. Thus, out of 44 patients studied, 19 patients had a TUG value $<15$ seconds and 25 patients had a TUG value over 15 seconds.

It was observed that patients with a higher TUG value (> 15 seconds) versus patients with normal TUG value (< 15 seconds) were older (70.92 years $+/-9.729$ vs. 66.89 years $+/-11.362 p=0.10)$, had a higher creatinine value (1.3435 mg/dl +/- 0.71260 vs. $1.0618 \mathrm{mg} / \mathrm{dl}$ $+/-0.33332 \mathrm{p}=0.896)$ and a higher blood glucose value (115.44 +/- 52.041 vs. 106.87 +/- $26.686 p=0.615)$.

After applying the treatment of heart failure during hospitalization, an improvement in walking speed was observed in patients, but not an improvement in TUG value.

We did not find any significant correlation between walking speed and the cardiac ultrasound parameters analyzed.

There were no adverse events during the functional capacity assessment with G-walk test.

\section{DISCUSSION}

The use of the G-Walk device for measuring walking speed and TUG is a safe method in the analysis of func- tional capacity in patients with cardiovascular pathology, in order to establish the medical rehabilitation plan. The G-Walk device is a safe and easy method to implement both in the hospital and in the outpatient setting, saving time and money regarding the low costs.

In our study, walking speed and TUG were not significantly correlated with cardiovascular risk factors present in our patients: hypertension, type II diabetes, smoking, dyslipidemia, unlike other studies in the literature. A possible explanation would be the younger age of our studied group and the relatively low number of the patients.

Studies in the literature have shown that elderly patients with chronic kidney disease (CKD) are associated with low mobility and decreased walking speed $(7,8)$. The HEALTH ABC study showed that adults older than 70 years, with associated CKD are prone to decreased mobility after 4.5 years of follow-up (9).

Older age, female sex, low body mass index, anemia, increased heart rate at rest and diabetes have been correlated to decreased six minutes walking distance (6 MWD) in patients with HF in various studies; similar findings were reported in patients without HF $(10,11)$.

During hospitalization, after the specific treatment of heart failure, an improvement in walking speed was observed. In the literature, the improvement of walking speed after the initiation of specific treatment has been associated with a favorable long-term prognosis (12).

The TUG test is a safe and easy-to-use method of measuring functional mobility in both cardiac and pulmonary patients such as chronic obstructive pulmonary disease (COPD). In some studies, the TUG tests results were associated with a great risk for falls and fractures, disability resulting in a low quality of life $(13,14)$.

To our knowlegde, in the literature studies were limited to a specific population (eg. men, CKD). Howerer in a large longitufinal study conducted in an Asian country evaluated the relationship between TUG and future incidence of cardiovascular (CV) event and mortality (14).

The strengths of the study are: the association of risk factors such as age, diabetes, chronic kidney disease with low mobility in patients with heart failure. Low long-term mobility increases the risk of cardiovascular events and thus increases mortality.

The limit of the study is the fact that the groups had a small number of patients, which limited our statistical power.

\section{CONCLUSIONS}

Following the study of the 44 patients with NYHA class II-III ICC, using the G-Walk device, it was found 
that patients with lower walking speed $(<0.8 \mathrm{~m} / \mathrm{s})$ are older, have a lower body weight, a lower value of oxygen saturation, a lower value of sodium and a higher value of glycemia.

It was also observed that patients with an increased value of the Timed Up and Go test are older, have a higher value of creatinine and glucose.

Thus, we can conclude that risk factors such as diabetes, chronic kidney disease, old age and the presence of heart failure are associated with low mobility.
Low mobility increases the risk of cardiovascular disease, thus increasing long-term morbidity and mortality.

We can thus conclude that the implementation of physical rehabilitation programs to increase mobility in patients with heart failure, along with drug treatment and the correction of risk factors is essential in reducing morbidity and mortality due to cardiovascular diseTable 2ase.

Conflict of interest: none declared Financial support: none declared

\section{REFERENCES}

1. Hardy SE, Perera S, Roumani YF, Chandler JM, Studenski SA. Improvement in usual gait speed predicts better survival in older adults. J Am Geriatr Soc. 2007 Nov;55(11):1727-34.

2. Ponikowski P, Voors AA, Anker SD, Bueno $\mathrm{H}$, Cleland JGF, et al.; ESC Scientific Document Group. 2016 ESC Guidelines for the diagnosis and treatment of acute and chronic heart failure: The Task Force for the diagnosis and treatment of acute and chronic heart failure of the European Society of Cardiology (ESC) Developed with the special contribution of the Heart Failure Association (HFA) of the ESC. Eur Heart $J$. 2016 Jul 14;37(27):2129-2200.

3. Perk J, De Backer G, Gohlke H, Graham I, Reiner Z, et al.; European Association for Cardiovascular Prevention \& Rehabilitation (EACPR); ESC Committee for Practice Guidelines (CPG). European Guidelines on cardiovascular disease prevention in clinical practice (version 2012). The Fifth Joint Task Force of the European Society of Cardiology and Other Societies on Cardiovascular Disease Prevention in Clinical Practice (constituted by representatives of nine societies and by invited experts). Eur Heart J. 2012 Jul;33(13):1635-701.

4. Remme WJ, Swedberg K; European Society of Cardiology. Comprehensive guidelines for the diagnosis and treatment of chronic heart failure. Task force for the diagnosis and treatment of chronic heart failure of the
European Society of Cardiology. Eur $\mathrm{J}$ Heart Fail. 2002 Jan;4(1):11-22.

5. Giannitsi S, Bougiakli M, Bechlioulis A, Kotsia A, Michalis LK, Naka KK. 6-minute walking test: a useful tool in the management of heart failure patients. Ther Adv Cardiovasc Dis. 2019 JanDec;13:1753944719870084.

6. Hwang R, Morris NR, Mandrusiak A, Mudge A, Suna J, Adsett J, Russell T. Timed Up and Go Test: A Reliable and Valid Test in Patients With Chronic Heart Failure. J Card Fail. 2016 Aug;22(8):646-50.

7. Liu CK, Lyass A, Massaro JM, D’Agostino RB Sr, Fox CS, Murabito JM. Chronic kidney disease defined by cystatin $C$ predicts mobility disability and changes in gait speed: the Framingham Offspring Study. J Gerontol A Biol Sci Med Sci. 2014 Mar;69(3):301-7.

8. Fried LF, Lee JS, Shlipak M, Chertow GM, Green C, Ding J, Harris T, Newman AB. Chronic kidney disease and functional limitation in older people: health, aging and body composition study. J Am Geriatr Soc. 2006 May;54(5):750-6.

9. Madero M, Peralta C, Katz R, Canada R, Fried L, Najjar S, et al.; Health ABC Study. Association of arterial rigidity with incident kidney disease and kidney function decline: the Health ABC study. Clin J Am Soc Nephrol. 2013 Mar;8(3):424-33.

10. Wanderley FA, Oliveira J, Mota J, Carvalho MJ. Six-minute walk distance (6MWD) is associated with body fat, systolic blood pressure, and rate-pressure product in community dwelling elderly subjects. Arch Gerontol Geriatr. 2011 MarApr;52(2):206-10.

11. Chun SH, Cho B, Yang HK, Ahn E, Han MK, Oh B, Shin DW, Son KY. Performance on physical function tests and the risk of fractures and admissions: Findings from a national health screening of 557,648 community-dwelling older adults. Arch Gerontol Geriatr. 2017 Jan-Feb;68:174-180.

12. Donoghue OA, Savva GM, Cronin H, Kenny RA, Horgan NF. Using timed up and go and usual gait speed to predict incident disability in daily activities among community-dwelling adults aged 65 and older. Arch Phys Med Rehabil. 2014 Oct;95(10):1954-61.

13. Ekström H, Dahlin-Ivanoff $S$, Elmståhl S. Effects of walking speed and results of timed get-up-and-go tests on quality of life and social participation in elderly individuals with a history of osteoporosis-related fractures. J Aging Health. 2011 Dec;23(8):1379-99.

14. Son KY, Shin DW, Lee JE, Kim SH, Yun JM, Cho B. Association of timed up and go test outcomes with future incidence of cardiovascular disease and mortality in adults aged 66 years: Korean national representative longitudinal study over 5.7 years. BMC Geriatr. 2020 Mar 19;20(1):111. 